\title{
Wheat Heat Tolerance: Mechanism, Impact and Quantitative Trait Loci Associated with Heat Tolerance
}

\author{
Anand Kumar ${ }^{1 *}$ and Vivudh Pratap Singh ${ }^{2}$ \\ ${ }^{1}$ Department of Genetics and Plant Breeding, Chandra Shekhar Azad University of \\ Agriculture and Technology, Kanpur (Uttar Pradesh), 208002, India \\ ${ }^{2}$ Department of Genetics and Plant Breeding, Chaudhary Charan Singh University, \\ Meerut, (Uttar Pradesh), 250004, India \\ *Corresponding author
}

\section{A B S T R A C T}

Wheat (Triticum aestivum L.) is one of the important cereal crops. It has the top first rank in among cereals because of which it has most significant

\section{Keywords}

Higher biochemical temperature

breeding

Article Info

Accepted:

xx June 2020

Available Online:

xx July 2020 contribution to the worldwide. Now day's high temperature is continuing raising that affected to wheat crop. This temperature is risen by human activities and some other activities. Heat stress is an abiotic phenomenon that resulting the losses in yield. High temperature alters the primary phenomena such as growth and development and also affect to the physiological responses and biochemical aspects. So we need to develop heat tolerance variety that can give good yield performance during heat stress. This may be achieved by the knowledge of whole plant mechanism such as mechanism of heat tolerance with plants, morphological responses, anatomical responses, physiological responses and expression of gene. Now days most of the tool to be adopted is molecular marker to know the idea about mechanism of heat stress. Molecular marker system reveals idea about which one gene/ QTLs associated with heat tolerance.

\section{Introduction}

Wheat (Triticum aestivum L.) is one of the most important Rabi cereals crop in all over the world. It is a thermo stable crop that is cultivated on latitudinal distribution (Sahu et al., 2002). Wheat is consumed by the human in all tropical and subtropical areas in developing as well as developed nations. It grows mainly $15-18^{0} \mathrm{C}$ optimum temperature (Chaudhary and wardlaw, 1978). But above temperature from particular temperature, it suffers the chronic heat tolerance during reproductive phase and vegetative growth. Main causes of high temperature are the global warming (Iba, 2002). Heat stress affected to the production of wheat in arid and semi-arid areas. So to consider these problems 
we need to develop heat tolerance variety for public domain. Genetic diversity for wheat heat tolerance has been well created (AlKhatib and Paulsen, 1990; Reynolds et al., 2009). If temperature rose from optimal temperature then this temperature reduces to wheat yield (Fokar et al., 1998; Maestri et al., 2002). There are many traits such as earliness, leaf rolling, leaf motif, plant height and grain filling duration has been associated with wheat heat tolerance (Blum et al.,1997, Fokar et al., 1998; Reynolds et al., 2009). There are many physiological traits associated with wheat heat resistance such as photosynthetic rate, stomata conductance and thermal stability (Al-Khatib and Paulsen, 1984; Fokar et al.,1998; Reynolds et al., 2009). High temperature at this time is produced severe loss to wheat production over worldwide (Hays et al., 2007).

Heat changes the morphological and anatomical structure of wheat plants resulting losses in yield (Stone and Nicolas, 1995). Physiological basis of wheat plants decide to select better genotype during high stress, this approach is very helpful to decide the tolerated variety. But this point should be always note that a variety gives good performance in heat tolerance, than we cannot consider that variety will give good yield performance under drought tolerance (Reynolds et al., 2009). Hence there is any other method need to predict the heat resistance variety such as marker assisted breeding/ marker assisted selection (MAS/MAB). Hence need the QTLs mapping to detect QTLs that is associated with heat resistance with the help of molecular markers (Kato et al., 2000). It is proved that heat stress is controlled by the quantitative nature and it is inherited from generation to generation (Blum et al.,1989; Yang et al., 2002). To detect the QTLs for heat tolerance is being by many plant breeders in different environment breeding programmes on different population (See table).
The main objective of QTLs mapping to detect the quantitative trait associated with heat tolerance with the help of molecular marker breeding (Mohammadi et al., 2008). There are many QTLs have been detected with the help molecular marker. They are described below (See table No. 01).

\section{Mechanism of heat tolerance}

When temperature is raised beyond level of threshold for a limited time, it can be reduced to yield. For higher yield, temperature should be normal range $18-22^{\circ} \mathrm{C}$. High temperature changed the physiological, morphological, anatomical and biochemical aspects of plants. High temperature must be change the plant anatomy in which plants can be mature in early stage (Porter, 2005). High temperature leads the injury levels of cellular that may be death of cells. Direct injuries of cells due to heat leads protein denaturation and also may be alter the mechanism of cell, if there is minimum temperature than it can be also inactive of enzymes and reduce the protein synthesis (Howarth, 2005). All these injuries leads to starvation death of cells in which these injuries directly affected to yield contribution characters (Smertenko et al., 1997). Plants are affected in different ways such as high temperatures and low temperature and high soil temperatures. In addition there are many crops that are very sensitive to heat temperatures. In general heat tolerance is one phenomenon in which plant can be maintaining self-integrity to avoid the high temperatures and can be create good metabolic pathway.

\section{Morphological response}

Pre and post-harvest losses has been created by heat stress. In which there are some morphological responses involving such as sunburn on leaves, stem and branches, scorching of leaves, leaf abscission and 
senescence, inhibition of root, shoot growth, fruit dropping, discoloration, damage etc. thus resulting reduced in yield (Vollenweider et al., 2005). And higher temperature also alters the phonological process from one to another. To enhance duration of high temperature decline the germination process or may be lost germination ultimately, this temperature inhibit the growth of emergence. Several plants have been affected by the high temperature in which significant reduction in yield, net assimilation ratio and shoot dry mass. It also affected to anthesis and reproduction stage of plants with the combination of heat stress and drought stress. For instances, both grain number and grain weight is also affected by the high temperature in wheat (Ferris et al., 1998). Reproductive process also affected by the higher temperature because of which fertilization can occur or not.

\section{Anatomical response}

It is clarify that high temperature not only affected to plants morphology at cellular level but also affected to subcellular level. This phenomenon is happened as same as in drought stress environment. Hence plants reduced the cell size, closer the stomata, it also increase the stomata densities and other cellular activity and enhance the xylem vessels for shoot and root (Anon et al., 2004). The most disadvantage of plants alteration the subcellular level in chloroplasts because of which significant changes in photosynthesis, for instances photosynthesis reduced by the higher temperature by changing in structural system of thylakoid (Karim et al., 1997).

\section{Physiological responses}

High temperature on wheat, reduce the physiological responses such as reduce the spike length, lower no of spikelets, accelerate floral initiation, adversely affect on pollen development etc. the most critical period of grain filling is post anthesis, if at this time temperature is high can be reduce yield. Complete sterility may be developed on more than $30^{\circ} \mathrm{c}$ temperature (Oven 1971, Kumar et al., 2020; Saini and Aspinal 1982).

Previous study has been conducted where out length of vegetative growth having highly positive correlation association with no of spikelets per spike (Rehman et al., 1997). While shorter length of vegetative growth having adverse effect and reduce the no of spikelets per spike. Higher temperature during floral initiation reduces the kernel number in plants. It is suggested that wheat yield and wheat quality could be improved to give heat shock treatment in early grain filling stage.

\section{Molecular approach}

It has been described that there are many methods to improve the heat tolerance genotype such as traditional, transgenic approach and conventional breeding approach. It is well suggested that heat tolerance is controlled by the multigenic trait that involve in gene expression at different level of stage in different tissue (Bohnert et al., 2006).

Thus to know about the heat tolerance gene, we need some extra powerful tool to detect the heat tolerance genes. The most powerful tool to detect the qualitative and quantitative complex traits is marker system (Roff, 1997. Shah et al.,1999; Kumar et al., 2020).

There are many marker system has been promising to detect the QTL. There are many markers that has been most extensively utilized in plant breeding to detect the heat tolerance QTLs but in this review paper we will discuss some markers (Table No. 01) is briefly described here. 
Table.1 Schematic representation of Quantitative trait loci with heat tolerance

\begin{tabular}{|c|c|c|c|c|c|c|c|}
\hline Trait & QTL & $\begin{array}{l}\text { Chromoso } \\
\text { me No. }\end{array}$ & Marker interval & $\begin{array}{l}\text { Populati } \\
\text { on Type }\end{array}$ & $\begin{array}{l}\text { Populati } \\
\text { on Size }\end{array}$ & Cross & References \\
\hline Grain Yield & QYld.1A & $1 \mathrm{~A}$ & $\begin{array}{c}\text { wPt-668205-wPt- } \\
731282\end{array}$ & RIL & 186 & Roshan $\times$ Superhead 2 & $\begin{array}{l}\text { Zandipour et al., } \\
\text { (2020) }\end{array}$ \\
\hline Grain Yield & QYld.1B & $1 \mathrm{~B}$ & wPt-3465-wPt-5801 & RIL & 186 & Roshan $\times$ Superhead 2 & $\begin{array}{l}\text { Zandipour et al., } \\
\text { (2020) }\end{array}$ \\
\hline Grain Yield & QYld.3B & $3 B$ & $\begin{array}{c}\text { wPt-667607-wPt- } \\
666139\end{array}$ & RIL & 186 & Roshan $\times$ Superhead 2 & $\begin{array}{l}\text { Zandipour et al., } \\
(2020)\end{array}$ \\
\hline Grain Yield & QYld.2D & $2 \mathrm{D}$ & $\begin{array}{c}\text { wPt-729831-wPt- } \\
730613\end{array}$ & RIL & 186 & Roshan $\times$ Superhead 2 & $\begin{array}{l}\text { Zandipour et al., } \\
\text { (2020) }\end{array}$ \\
\hline $\begin{array}{l}\text { Biological } \\
\text { yield }\end{array}$ & QByld.4A & $4 \mathrm{~A}$ & wPt-2084-wPt-671844 & RIL & 186 & Roshan $\times$ Superhead 2 & $\begin{array}{l}\text { Zandipour et al., } \\
\text { (2020) }\end{array}$ \\
\hline $\begin{array}{c}\text { Biological } \\
\text { yield }\end{array}$ & QByld.1B & $1 \mathrm{~B}$ & wPt-3465-wPt-5801 & RIL & 186 & Roshan $\times$ Superhead 2 & $\begin{array}{l}\text { Zandipour et al., } \\
(2020)\end{array}$ \\
\hline $\begin{array}{l}\text { Biological } \\
\text { yield }\end{array}$ & QByld.3B & $3 B$ & wPt-729904-gwm247 & RIL & 186 & Roshan $\times$ Superhead 2 & $\begin{array}{l}\text { Zandipour et al., } \\
\text { (2020) }\end{array}$ \\
\hline $\begin{array}{l}\text { Thousand } \\
\text { grain weight }\end{array}$ & $Q T g w .1 B$ & $1 \mathrm{~B}$ & wPt-5801-wPt-2019 & RIL & 186 & Roshan $\times$ Superhead 2 & $\begin{array}{l}\text { Zandipour et al., } \\
\text { (2020) }\end{array}$ \\
\hline $\begin{array}{l}\text { Thousand } \\
\text { grain weight }\end{array}$ & $Q T g w .2 B_{1}$ & $2 \mathrm{~B}$ & Cfd73-wPt-8460 & RIL & 186 & Roshan $\times$ Superhead 2 & $\begin{array}{l}\text { Zandipour et al., } \\
(2020)\end{array}$ \\
\hline $\begin{array}{l}\text { Thousand } \\
\text { grain weight }\end{array}$ & $Q T g w .2 B_{2}$ & $2 \mathrm{~B}$ & wPt-4301-wPt-3132 & RIL & 186 & Roshan $\times$ Superhead 2 & $\begin{array}{l}\text { Zandipour et al., } \\
(2020)\end{array}$ \\
\hline $\begin{array}{l}\text { Thousand } \\
\text { grain weight }\end{array}$ & $Q T g w .3 B$ & $3 B$ & wPt-1484-gwm566 & RIL & 186 & Roshan $\times$ Superhead 2 & $\begin{array}{l}\text { Zandipour et al., } \\
\text { (2020) }\end{array}$ \\
\hline $\begin{array}{c}\text { Grain } \\
\text { number per } \\
\text { spike }\end{array}$ & QGnи.6A & $6 \mathrm{~A}$ & gwm334-wPt-7330 & RIL & 186 & Roshan $\times$ Superhead 2 & $\begin{array}{l}\text { Zandipour et al., } \\
(2020)\end{array}$ \\
\hline $\begin{array}{c}\text { Grain } \\
\text { number per }\end{array}$ & $Q G n u .7 A$ & $7 \mathrm{~A}$ & gwm635-wPt-4877 & RIL & 186 & Roshan $\times$ Superhead 2 & $\begin{array}{l}\text { Zandipour et al., } \\
(2020)\end{array}$ \\
\hline
\end{tabular}




\begin{tabular}{|c|c|c|c|c|c|c|c|}
\hline spike & & & & & & & \\
\hline $\begin{array}{c}\text { Grain } \\
\text { number per } \\
\text { spike }\end{array}$ & $Q G n u .1 B$ & $1 \mathrm{~B}$ & wPt-3465-wPt-5801 & RIL & 186 & Roshan $\times$ Superhead 2 & $\begin{array}{l}\text { Zandipour et al., } \\
(2020)\end{array}$ \\
\hline $\begin{array}{c}\text { Grain } \\
\text { number per } \\
\text { spike }\end{array}$ & $Q G n u .2 B_{3}$ & $2 \mathrm{~B}$ & wPt-5795-wPt-2106 & RIL & 186 & Roshan $\times$ Superhead 2 & $\begin{array}{l}\text { Zandipour et al., } \\
(2020)\end{array}$ \\
\hline $\begin{array}{c}\text { Grain } \\
\text { number per } \\
\text { spike }\end{array}$ & $Q G n u .3 B$ & $3 B$ & wPt-666139-wPt-4412 & RIL & 186 & Roshan $\times$ Superhead 2 & $\begin{array}{l}\text { Zandipour et al., } \\
(2020)\end{array}$ \\
\hline $\begin{array}{c}\text { Grain } \\
\text { number per } \\
\text { spike }\end{array}$ & $Q G n u .6 B_{2}$ & $6 \mathrm{~B}$ & wPt-3378-wPt-6282 & RIL & 186 & Roshan $\times$ Superhead 2 & $\begin{array}{l}\text { Zandipour et al., } \\
(2020)\end{array}$ \\
\hline $\begin{array}{c}\text { Grain } \\
\text { number per } \\
\text { spike }\end{array}$ & $Q G n u .7 B$ & $7 \mathrm{~B}$ & wPt-9326-wPt-7975 & RIL & 186 & Roshan $\times$ Superhead 2 & $\begin{array}{l}\text { Zandipour et al., } \\
(2020)\end{array}$ \\
\hline $\begin{array}{c}\text { Grain } \\
\text { number per } \\
\text { spike }\end{array}$ & $Q G n u .1 D$ & $1 \mathrm{D}$ & wPt-1799-wPt-3707 & RIL & 186 & Roshan $\times$ Superhead 2 & $\begin{array}{l}\text { Zandipour et al., } \\
(2020)\end{array}$ \\
\hline Spike length & $Q S l .7 A$ & $7 \mathrm{~A}$ & wPt-4877-wPt-6217 & RIL & 186 & Roshan $\times$ Superhead 2 & $\begin{array}{l}\text { Zandipour et al., } \\
(2020)\end{array}$ \\
\hline Spike length & $Q S l .1 B$ & $1 \mathrm{~B}$ & wPt-3465-wPt-5801 & RIL & 186 & Roshan $\times$ Superhead 2 & $\begin{array}{l}\text { Zandipour et al., } \\
(2020)\end{array}$ \\
\hline Spike length & QSl.6B & $6 \mathrm{~B}$ & wPt-663764wPt-7954 & RIL & 186 & Roshan $\times$ Superhead 2 & $\begin{array}{l}\text { Zandipour et al., } \\
(2020)\end{array}$ \\
\hline $\begin{array}{c}\text { Spike } \\
\text { weight }\end{array}$ & $Q S w .1 B$ & $1 \mathrm{~B}$ & wPt-3465-wPt-5801 & RIL & 186 & Roshan $\times$ Superhead 2 & $\begin{array}{l}\text { Zandipour et al., } \\
(2020)\end{array}$ \\
\hline $\begin{array}{l}\text { Spike } \\
\text { weight }\end{array}$ & $Q S w .2 B$ & $2 \mathrm{~B}$ & Cfd73-wPt-8460 & RIL & 186 & Roshan $\times$ Superhead 2 & $\begin{array}{l}\text { Zandipour et al., } \\
(2020)\end{array}$ \\
\hline $\begin{array}{l}\text { Spike } \\
\text { weight }\end{array}$ & $Q S w .3 B$ & $3 \mathrm{~B}$ & wPt-5358-wPt-5390 & RIL & 186 & Roshan $\times$ Superhead 2 & $\begin{array}{l}\text { Zandipour et al., } \\
(2020)\end{array}$ \\
\hline
\end{tabular}




\begin{tabular}{|c|c|c|c|c|c|c|c|}
\hline $\begin{array}{c}\text { Spike } \\
\text { weight }\end{array}$ & $Q S w .2 D$ & $2 \mathrm{D}$ & $\begin{array}{c}\text { wPt-729831-wPt- } \\
730613\end{array}$ & RIL & 186 & Roshan $\times$ Superhead 2 & $\begin{array}{l}\text { Zandipour et al., } \\
\text { (2020) }\end{array}$ \\
\hline $\begin{array}{c}\text { Grain } \\
\text { number per } \\
\text { spike }\end{array}$ & QSpn.4A-1 & $4 \mathrm{~A}$ & $\begin{array}{c}\text { wPt-665730-wPt- } \\
665927\end{array}$ & RIL & 186 & Roshan $\times$ Superhead 2 & $\begin{array}{l}\text { Zandipour et al., } \\
\text { (2020) }\end{array}$ \\
\hline $\begin{array}{c}\text { Grain } \\
\text { number per } \\
\text { spike }\end{array}$ & QSpn.4A-2 & $4 \mathrm{~A}$ & $\begin{array}{c}\text { wPt-664047-wPt- } \\
669103\end{array}$ & RIL & 186 & Roshan $\times$ Superhead2 & $\begin{array}{l}\text { Zandipour et al., } \\
\text { (2020) }\end{array}$ \\
\hline $\begin{array}{c}\text { Grain } \\
\text { number per } \\
\text { spike }\end{array}$ & QSpn.5A & $5 \mathrm{~A}$ & wPt-665622-wPt-1954 & RIL & 186 & Roshan $\times$ Superhead 2 & $\begin{array}{l}\text { Zandipour et al., } \\
\text { (2020) }\end{array}$ \\
\hline $\begin{array}{c}\text { Grain } \\
\text { number per } \\
\text { spike }\end{array}$ & QSpn.1B & $1 \mathrm{~B}$ & wPt-5801-wPt-2019 & RIL & 186 & Roshan $\times$ Superhead 2 & $\begin{array}{l}\text { Zandipour et al., } \\
\text { (2020) }\end{array}$ \\
\hline $\begin{array}{c}\text { Grain } \\
\text { number per } \\
\text { spike }\end{array}$ & QSpn.3B-1 & $3 B$ & wPt-5390-wPt-669328 & RIL & 186 & Roshan $\times$ Superhead 2 & $\begin{array}{l}\text { Zandipour et al., } \\
\text { (2020) }\end{array}$ \\
\hline $\begin{array}{c}\text { Grain } \\
\text { number per } \\
\text { spike }\end{array}$ & QSpn.3B-2 & $3 B$ & $\begin{array}{c}\text { wPt-731120-wPt- } \\
664771\end{array}$ & RIL & 186 & Roshan $\times$ Superhead 2 & $\begin{array}{l}\text { Zandipour et al., } \\
(2020)\end{array}$ \\
\hline $\begin{array}{c}\text { Grain } \\
\text { number per } \\
\text { spike }\end{array}$ & QSpn.5B & $5 B$ & wPt-3457-wPt-3661 & RIL & 186 & Roshan $\times$ Superhead 2 & $\begin{array}{l}\text { Zandipour et al., } \\
\text { (2020) }\end{array}$ \\
\hline $\begin{array}{c}\text { Grain } \\
\text { number per } \\
\text { spike }\end{array}$ & QSpn.7B & $7 \mathrm{~B}$ & wPt-2025-wPt-7894 & RIL & 186 & Roshan $\times$ Superhead 2 & $\begin{array}{l}\text { Zandipour et al., } \\
(2020)\end{array}$ \\
\hline $\begin{array}{c}\text { Grain } \\
\text { number per } \\
\text { spike }\end{array}$ & QSpn.5D & $5 \mathrm{D}$ & wPt-671760-wPt-5505 & RIL & 186 & Roshan $\times$ Superhead2 & $\begin{array}{l}\text { Zandipour et al., } \\
\text { (2020) }\end{array}$ \\
\hline $\begin{array}{c}\text { Grain } \\
\text { number per } \\
\text { spike }\end{array}$ & QSpn.7D & $7 \mathrm{D}$ & $\begin{array}{c}\text { wPt-664088-wPt- } \\
667257\end{array}$ & RIL & 186 & Roshan $\times$ Superhead 2 & $\begin{array}{l}\text { Zandipour et al., } \\
(2020)\end{array}$ \\
\hline
\end{tabular}




\begin{tabular}{|c|c|c|c|c|c|c|c|}
\hline Stem weight & $Q S t w \cdot 1 A_{1}$ & $1 \mathrm{~A}$ & $\begin{array}{c}\text { wPt-665590-wPt- } \\
672158\end{array}$ & RIL & 186 & Roshan $\times$ Superhead 2 & $\begin{array}{l}\text { Zandipour et al., } \\
\text { (2020) }\end{array}$ \\
\hline Stem weight & QStw.4A & $4 \mathrm{~A}$ & wPt-2084-wPt-671844 & RIL & 186 & Roshan $\times$ Superhead 2 & $\begin{array}{l}\text { Zandipour et al., } \\
\text { (2020) }\end{array}$ \\
\hline Stem weight & QStw.1B & $1 \mathrm{~B}$ & wPt-5801-wPt-2019 & RIL & 186 & Roshan $\times$ Superhead2 & $\begin{array}{l}\text { Zandipour et al., } \\
\text { (2020) }\end{array}$ \\
\hline Stem weight & $Q S t w \cdot 2 B_{2}$ & $2 \mathrm{~B}$ & wPt-3132-wPt-0950 & RIL & 186 & Roshan $\times$ Superhead 2 & $\begin{array}{l}\text { Zandipour et al., } \\
\text { (2020) }\end{array}$ \\
\hline Stem weight & $Q S t w .3 B$ & $3 B$ & gwm247-wPt-667324 & RIL & 186 & Roshan $\times$ Superhead 2 & $\begin{array}{l}\text { Zandipour et al., } \\
\text { (2020) }\end{array}$ \\
\hline Stem weight & QStw.7B & $7 \mathrm{~B}$ & wPt-2305-wPt-1149 & RIL & 186 & Roshan $\times$ Superhead 2 & $\begin{array}{l}\text { Zandipour et al., } \\
\text { (2020) }\end{array}$ \\
\hline Stem weight & $Q S t w .1 D$ & $1 \mathrm{D}$ & wPt-9664-wPt-7437 & RIL & 186 & Roshan $\times$ Superhead 2 & $\begin{array}{l}\text { Zandipour et al., } \\
\text { (2020) }\end{array}$ \\
\hline Plant height & $Q H t .1 B$ & 1B & $w P t-5801-w P t-2019$ & RIL & 186 & Roshan $\times$ Superhead 2 & $\begin{array}{l}\text { Zandipour et al., } \\
\text { (2020) }\end{array}$ \\
\hline $\begin{array}{l}\text { Peduncle } \\
\text { length }\end{array}$ & $Q P d l .1 A_{1}$ & $1 \mathrm{~A}$ & $\begin{array}{c}\text { wPt-665590-wPt- } \\
672158\end{array}$ & RIL & 186 & Roshan $\times$ Superhead2 & $\begin{array}{l}\text { Zandipour et al., } \\
(2020)\end{array}$ \\
\hline $\begin{array}{l}\text { Peduncle } \\
\text { length }\end{array}$ & $Q P d l .3 B$ & $3 B$ & wPt-666139-wPt-4412 & RIL & 186 & Roshan $\times$ Superhead 2 & $\begin{array}{l}\text { Zandipour et al., } \\
\text { (2020) }\end{array}$ \\
\hline $\begin{array}{l}\text { Peduncle } \\
\text { length }\end{array}$ & $Q P d l .2 D_{l}$ & $2 \mathrm{D}$ & wPt-9848-wPt-3757 & RIL & 186 & Roshan $\times$ Superhead 2 & $\begin{array}{l}\text { Zandipour et al., } \\
\text { (2020) }\end{array}$ \\
\hline $\begin{array}{l}\text { Peduncle } \\
\text { length }\end{array}$ & $Q P d l .7 D$ & $7 \mathrm{D}$ & wPt-4254-wPt-672113 & RIL & 186 & Roshan $\times$ Superhead 2 & $\begin{array}{l}\text { Zandipour et al., } \\
\text { (2020) }\end{array}$ \\
\hline $\begin{array}{l}\text { Node } \\
\text { number }\end{array}$ & $Q N n .2 B 2$ & $2 \mathrm{~B}$ & $w P t-4301-w P t-3132$ & RIL & 186 & Roshan $\times$ Superhead 2 & $\begin{array}{l}\text { Zandipour et al., } \\
\text { (2020) }\end{array}$ \\
\hline $\begin{array}{c}\text { Node } \\
\text { number }\end{array}$ & $Q N n .3 B$ & $3 B$ & $\begin{array}{c}w P t-666964-w P t- \\
731524\end{array}$ & RIL & 186 & Roshan $\times$ Superhead2 & $\begin{array}{l}\text { Zandipour et al., } \\
(2020)\end{array}$ \\
\hline $\begin{array}{c}\text { Node } \\
\text { number }\end{array}$ & $Q N n .6 B 2$ & $6 B$ & $w P t-6286-w P t-9532$ & RIL & 186 & Roshan $\times$ Superhead 2 & $\begin{array}{l}\text { Zandipour et al., } \\
(2020)\end{array}$ \\
\hline $\begin{array}{c}\text { Node } \\
\text { number }\end{array}$ & $Q N n .2 D 1$ & $2 \mathrm{D}$ & $\begin{array}{c}w P t-667584-w P t- \\
671990\end{array}$ & RIL & 186 & Roshan $\times$ Superhead 2 & $\begin{array}{l}\text { Zandipour et al., } \\
\text { (2020) }\end{array}$ \\
\hline
\end{tabular}




\begin{tabular}{|c|c|c|c|c|c|c|c|}
\hline $\begin{array}{c}\text { Node } \\
\text { number }\end{array}$ & $Q N n .7 D$ & 7D & $\begin{array}{c}w P t-664438-w P t- \\
663984\end{array}$ & RIL & 186 & Roshan $\times$ Superhead2 & $\begin{array}{l}\text { Zandipour et al., } \\
\text { (2020) }\end{array}$ \\
\hline $\begin{array}{l}\text { HSI Canopy } \\
\text { Temperatur } \\
\text { e }\end{array}$ & $\begin{array}{l}\text { QHCt.bhu- } \\
\quad \text { IDS }\end{array}$ & $1 \mathrm{D}$ & $w P t 9664-c f d 083$ & & & & Chandra et al., (2017) \\
\hline $\begin{array}{l}\text { Chlorophyll } \\
\text { content }\end{array}$ & - & - & Barc68 & RIL & 142 & Chirya $3 \times$ Sonalika & Kumar et. al. (2012) \\
\hline $\begin{array}{c}\text { Canopy } \\
\text { temperature }\end{array}$ & - & - & Barc101 & RIL & 142 & Chirya $3 \times$ Sonalika & Kumar et. al. (2012) \\
\hline Grain yield & - & - & Gwm190 & RIL & 144 & Kauz $\times$ MTRWA116 & $\begin{array}{l}\text { Mohammadi et. al. } \\
\text { (2008) }\end{array}$ \\
\hline $\begin{array}{l}\text { Flowering } \\
\text { (days) }\end{array}$ & QFlt.dms-2D & $2 \mathrm{D}$ & $\begin{array}{c}\text { Ppd-Dla- } \\
\text { wsnp_CAP11_c3842_18 } \\
29821\end{array}$ & RIL & 158 & Cutler $\times$ ACBarrie & $\begin{array}{l}\text { Perez-Lara et al., } \\
\text { (2016) }\end{array}$ \\
\hline $\begin{array}{l}\text { Flowering } \\
\text { (days) }\end{array}$ & QFlt.dms-2D & $2 \mathrm{D}$ & $\begin{array}{c}\text { Ppd-Dla- } \\
\text { wsnp_CAP11_c3842_18 } \\
\text { 29821 }\end{array}$ & RIL & 158 & Cutler $\times$ ACBarrie & $\begin{array}{l}\text { Perez-Lara et al., } \\
\text { (2016) }\end{array}$ \\
\hline $\begin{array}{l}\text { Flowering } \\
\text { (days) }\end{array}$ & QFlt.dms-2D & $2 \mathrm{D}$ & $\begin{array}{c}\text { Ppd-D1a- } \\
\text { wsnp_CAP11_c3842_18 } \\
\text { 29821 }\end{array}$ & RIL & 158 & Cutler $\times$ ACBarrie & $\begin{array}{l}\text { Perez-Lara et al., } \\
\text { (2016) }\end{array}$ \\
\hline $\begin{array}{l}\text { Flowering } \\
\text { (days) }\end{array}$ & QFlt.dms-2D & $2 \mathrm{D}$ & $\begin{array}{c}\text { wsnp_CAP11_c3842_18 } \\
29821\end{array}$ & RIL & 158 & Cutler $\times$ ACBarrie & $\begin{array}{l}\text { Perez-Lara et al., } \\
\text { (2016) }\end{array}$ \\
\hline $\begin{array}{l}\text { Flowering } \\
\text { (days) }\end{array}$ & QFlt.dms-2D & $2 \mathrm{D}$ & $\begin{array}{c}\text { Ppd-Dla- } \\
\text { wsnp_CAP11_c3842_18 } \\
29821\end{array}$ & RIL & 158 & Cutler $\times$ ACBarrie & $\begin{array}{l}\text { Perez-Lara et al., } \\
\text { (2016) }\end{array}$ \\
\hline $\begin{array}{l}\text { Flowering } \\
\text { (days) }\end{array}$ & QFlt.dms-2D & $2 \mathrm{D}$ & $\begin{array}{c}\text { Ppd-Dla- } \\
\text { wsnp_CAP11_c3842_18 } \\
29821\end{array}$ & RIL & 158 & Cutler $\times$ ACBarrie & $\begin{array}{l}\text { Perez-Lara et al., } \\
(2016)\end{array}$ \\
\hline $\begin{array}{l}\text { Flowering } \\
\text { (days) }\end{array}$ & QFlt.dms-2D & $2 \mathrm{D}$ & $\begin{array}{c}\text { Ppd-Dla- } \\
\text { wsnp_CAP11_c3842_18 } \\
\text { 29821 }\end{array}$ & RIL & 158 & Cutler $\times$ ACBarrie & $\begin{array}{l}\text { Perez-Lara et al., } \\
(2016)\end{array}$ \\
\hline
\end{tabular}




\begin{tabular}{|c|c|c|c|c|c|c|c|}
\hline $\begin{array}{l}\text { Flowering } \\
\text { (days) }\end{array}$ & QFlt.dms-2D & $2 \mathrm{D}$ & $\begin{array}{c}\text { wsnp_CAP11_c3842_18 } \\
29821\end{array}$ & RIL & 158 & Cutler $\times$ ACBarrie & $\begin{array}{l}\text { Perez-Lara et al., } \\
\text { (2016) }\end{array}$ \\
\hline $\begin{array}{l}\text { Flowering } \\
\text { (DD) }\end{array}$ & QFlt.dms-2D & $2 \mathrm{D}$ & $\begin{array}{c}\text { Ppd-Dla- } \\
\text { wsnp_CAP11_c3842_18 } \\
29821\end{array}$ & RIL & 158 & Cutler $\times$ ACBarrie & $\begin{array}{l}\text { Perez-Lara et al., } \\
(2016)\end{array}$ \\
\hline $\begin{array}{l}\text { Flowering } \\
\text { (DD) }\end{array}$ & QFlt.dms-2D & $2 \mathrm{D}$ & $\begin{array}{c}\text { wsnp_CAP11_c3842_18 } \\
29821\end{array}$ & RIL & 158 & Cutler $\times$ ACBarrie & $\begin{array}{l}\text { Perez-Lara et al., } \\
\text { (2016) }\end{array}$ \\
\hline $\begin{array}{l}\text { Flowering } \\
\text { (DD) }\end{array}$ & QFlt.dms-2D & $2 \mathrm{D}$ & $\begin{array}{c}\text { Ppd-Dla- } \\
\text { wsnp_CAP11_c3842_18 } \\
29821\end{array}$ & RIL & 158 & Cutler $\times$ ACBarrie & $\begin{array}{l}\text { Perez-Lara et al., } \\
(2016)\end{array}$ \\
\hline $\begin{array}{l}\text { Flowering } \\
\text { (DD) }\end{array}$ & QFlt.dms-2D & $2 \mathrm{D}$ & $\begin{array}{c}\text { Ppd-Dla- } \\
\text { wsnp_CAP11_c3842_18 } \\
29821\end{array}$ & RIL & 158 & Cutler $\times$ ACBarrie & $\begin{array}{l}\text { Perez-Lara et al., } \\
(2016)\end{array}$ \\
\hline $\begin{array}{l}\text { Flowering } \\
\text { (DD) }\end{array}$ & QFlt.dms-2D & $2 \mathrm{D}$ & $\begin{array}{c}\text { Ppd-Dla- } \\
\text { wsnp_CAP11_c3842_18 } \\
29821\end{array}$ & RIL & 158 & Cutler $\times$ ACBarrie & $\begin{array}{l}\text { Perez-Lara et al., } \\
(2016)\end{array}$ \\
\hline $\begin{array}{c}\text { Flowering } \\
\text { (days) }\end{array}$ & QFlt.dms-3B & $3 B$ & $\begin{array}{l}\text { Excalibur_c45968_83- } \\
\text { CAP12_rep_c7901_114 }\end{array}$ & RIL & 158 & Cutler $\times$ ACBarrie & $\begin{array}{l}\text { Perez-Lara et al., } \\
(2016)\end{array}$ \\
\hline $\begin{array}{l}\text { Flowering } \\
\text { (DD) }\end{array}$ & QFlt.dms-3B & $3 B$ & $\begin{array}{l}\text { Excalibur_c45968_83- } \\
\text { CAP12_rep_c7901_114 }\end{array}$ & RIL & 158 & Cutler $\times$ ACBarrie & $\begin{array}{l}\text { Perez-Lara et al., } \\
\text { (2016) }\end{array}$ \\
\hline $\begin{array}{l}\text { Flowering } \\
\text { (DD) }\end{array}$ & QFlt.dms-3B & $3 B$ & $\begin{array}{l}\text { Excalibur_c45968_83- } \\
\text { CAP12_rep_c7901_114 }\end{array}$ & RIL & 158 & Cutler $\times$ ACBarrie & $\begin{array}{l}\text { Perez-Lara et al., } \\
\text { (2016) }\end{array}$ \\
\hline $\begin{array}{l}\text { Flowering } \\
\text { (DD) }\end{array}$ & $\begin{array}{l}\text { QFlt.dms- } \\
\quad 4 A .1\end{array}$ & $4 \mathrm{~A}$ & $\begin{array}{c}\text { wsnp_Ex_c54453_5733 } \\
1510\end{array}$ & RIL & 158 & Cutler $\times$ ACBarrie & $\begin{array}{l}\text { Perez-Lara et al., } \\
\text { (2016) }\end{array}$ \\
\hline $\begin{array}{l}\text { Flowering } \\
\text { (days) }\end{array}$ & $\begin{array}{l}\text { QFlt.dms- } \\
\quad 4 A .1\end{array}$ & $4 \mathrm{~A}$ & $\begin{array}{c}\text { CAP12_rep_c4000_432 } \\
- \\
\text { wsnp_Ex_c54453_5733 } \\
1510\end{array}$ & RIL & 158 & Cutler $\times$ ACBarrie & $\begin{array}{l}\text { Perez-Lara et al., } \\
(2016)\end{array}$ \\
\hline $\begin{array}{l}\text { Flowering } \\
\text { (DD) }\end{array}$ & QFlt.dms $-4 A$ & $4 \mathrm{~A}$ & $\begin{array}{c}\text { CAP12_rep_c4000_432 } \\
- \\
\text { wsnp_Ex_c54453_5733 } \\
1510\end{array}$ & RIL & 158 & Cutler $\times$ ACBarrie & $\begin{array}{l}\text { Perez-Lara et al., } \\
(2016)\end{array}$ \\
\hline
\end{tabular}




\begin{tabular}{|c|c|c|c|c|c|c|c|}
\hline $\begin{array}{l}\text { Flowering } \\
\text { (days) }\end{array}$ & $\begin{array}{l}\text { QFlt.dms- } \\
\quad 4 A .2\end{array}$ & $4 \mathrm{~A}$ & $\begin{array}{c}\text { Ra_c7973_1185- } \\
\text { wsnp_Ex_c10390_1700 } \\
7929\end{array}$ & RIL & 158 & Cutler $\times$ ACBarrie & $\begin{array}{l}\text { Perez-Lara et al., } \\
(2016)\end{array}$ \\
\hline $\begin{array}{l}\text { Flowering } \\
\text { (DD) }\end{array}$ & $\begin{array}{l}\text { QFlt.dms- } \\
\quad 4 A .2\end{array}$ & $4 \mathrm{~A}$ & $\begin{array}{c}\text { Ra_c7973_1185- } \\
\text { wsnp_Ex_c10390_1700 } \\
7929\end{array}$ & RIL & 158 & Cutler $\times$ ACBarrie & $\begin{array}{l}\text { Perez-Lara et al., } \\
\text { (2016) }\end{array}$ \\
\hline $\begin{array}{l}\text { Flowering } \\
\text { (days) }\end{array}$ & $\begin{array}{l}\text { QFlt.dms- } \\
\quad 4 A .3\end{array}$ & $4 \mathrm{~A}$ & $\begin{array}{c}\text { BobWhite_c22176_295- } \\
\text { RAC875_c59673_500 }\end{array}$ & RIL & 158 & Cutler $\times$ ACBarrie & $\begin{array}{l}\text { Perez-Lara et al., } \\
(2016)\end{array}$ \\
\hline $\begin{array}{l}\text { Flowering } \\
\text { (DD) }\end{array}$ & $\begin{array}{l}\text { QFlt.dms- } \\
\quad 4 A .3\end{array}$ & $4 \mathrm{~A}$ & $\begin{array}{c}\text { BobWhite_c22176_295- } \\
\text { RAC875_c59673_500 }\end{array}$ & RIL & 158 & Cutler $\times$ ACBarrie & $\begin{array}{l}\text { Perez-Lara et al., } \\
\text { (2016) }\end{array}$ \\
\hline $\begin{array}{l}\text { Flowering } \\
\text { (days) }\end{array}$ & $\begin{array}{l}\text { QFlt.dms- } \\
\quad 5 A .1\end{array}$ & $5 \mathrm{~A}$ & $\begin{array}{c}\text { Kukri_c20258_143- } \\
J D \_c 3525 \_1503\end{array}$ & RIL & 158 & Cutler $\times$ ACBarrie & $\begin{array}{l}\text { Perez-Lara et al., } \\
(2016)\end{array}$ \\
\hline $\begin{array}{l}\text { Flowering } \\
\text { (DD) }\end{array}$ & $\begin{array}{l}\text { QFlt.dms- } \\
\quad 5 A .2\end{array}$ & $5 \mathrm{~A}$ & $\begin{array}{l}\text { Tdurum_contig86202_1 } \\
75- \\
\text { wsnp_Ra_c10915_1783 } \\
8202\end{array}$ & RIL & 158 & Cutler $\times$ ACBarrie & $\begin{array}{l}\text { Perez-Lara et al., } \\
(2016)\end{array}$ \\
\hline $\begin{array}{l}\text { Flowering } \\
\text { (DD) }\end{array}$ & $\begin{array}{l}\text { QFlt.dms- } \\
\quad 5 A .2\end{array}$ & $5 \mathrm{~A}$ & $\begin{array}{c}\text { wsnp_Ra_c12183_1958 } \\
7379- \\
\text { wsnp_Ra_c3414_63782 } \\
71\end{array}$ & RIL & 158 & Cutler $\times$ ACBarrie & $\begin{array}{l}\text { Perez-Lara et al., } \\
(2016)\end{array}$ \\
\hline $\begin{array}{c}\text { Flowering } \\
\text { (days) }\end{array}$ & QFlt.dms $-5 B$ & $5 B$ & $\begin{array}{c}\text { BS00063785_51- } \\
\text { IACX5818 }\end{array}$ & RIL & 158 & Cutler $\times$ ACBarrie & $\begin{array}{l}\text { Perez-Lara et al., } \\
\text { (2016) }\end{array}$ \\
\hline $\begin{array}{l}\text { Flowering } \\
\text { (days) }\end{array}$ & QFlt.dms-5B & $5 B$ & $\begin{array}{c}I A C X 5818- \\
\text { wsnp_Ku_c17875_2705 } \\
1169\end{array}$ & RIL & 158 & Cutler $\times$ ACBarrie & $\begin{array}{l}\text { Perez-Lara et al., } \\
(2016)\end{array}$ \\
\hline $\begin{array}{l}\text { Flowering } \\
\text { (days) }\end{array}$ & $\begin{array}{l}\text { QFlt.dms- } \\
\quad 6 B .1\end{array}$ & $6 B$ & $\begin{array}{c}\text { Tdurum_contig11700_1 } \\
247- \\
\text { wsnp_Ra_c2730_51903 } \\
65\end{array}$ & RIL & 158 & Cutler $\times$ ACBarrie & $\begin{array}{l}\text { Perez-Lara et al., } \\
\text { (2016) }\end{array}$ \\
\hline
\end{tabular}




\begin{tabular}{|c|c|c|c|c|c|c|c|}
\hline $\begin{array}{l}\text { Flowering } \\
\text { (days) }\end{array}$ & $\begin{array}{l}\text { QFlt.dms- } \\
\quad 6 B .1\end{array}$ & $6 \mathrm{~B}$ & $\begin{array}{l}\text { Tdurum_contig11700_1 } \\
\text { 247- } \\
\text { wsnp_Ra_c2730_51903 } \\
65\end{array}$ & RIL & 158 & Cutler $\times$ ACBarrie & $\begin{array}{l}\text { Perez-Lara et al., } \\
\text { (2016) }\end{array}$ \\
\hline $\begin{array}{l}\text { Flowering } \\
\text { (days) }\end{array}$ & $\begin{array}{l}\text { QFlt.dms- } \\
\quad 6 B .2\end{array}$ & $6 \mathrm{~B}$ & $\begin{array}{c}\text { wsnp_Ex_c4124_74552 } \\
\text { 25-Kukri_c49331_77 }\end{array}$ & RIL & 158 & Cutler $\times$ ACBarrie & $\begin{array}{l}\text { Perez-Lara et al., } \\
\text { (2016) }\end{array}$ \\
\hline $\begin{array}{l}\text { Flowering } \\
\text { (DD) }\end{array}$ & $\begin{array}{l}\text { QFlt.dms- } \\
\quad 6 B .2\end{array}$ & $6 \mathrm{~B}$ & $\begin{array}{c}\text { wsnp_Ex_c4124_74552 } \\
\text { 25-Kukri_c49331_77 }\end{array}$ & RIL & 158 & Cutler $\times$ ACBarrie & $\begin{array}{l}\text { Perez-Lara et al., } \\
\text { (2016) }\end{array}$ \\
\hline $\begin{array}{l}\text { Flowering } \\
\text { (DD) }\end{array}$ & $\begin{array}{l}\text { QFlt.dms- } \\
\quad \text { 6B.2 }\end{array}$ & $6 \mathrm{~B}$ & $\begin{array}{c}\text { wsnp_Ex_c4124_74552 } \\
\text { 25-Kukri_c49331_77 }\end{array}$ & RIL & 158 & Cutler $\times$ ACBarrie & $\begin{array}{l}\text { Perez-Lara et al., } \\
\text { (2016) }\end{array}$ \\
\hline $\begin{array}{c}\text { Flowering } \\
\text { (days) }\end{array}$ & QFlt.dms-7A & $7 \mathrm{~A}$ & $\begin{array}{c}\text { Excalibur_c16355_712- } \\
\text { RAC875_c18446_521 }\end{array}$ & RIL & 158 & Cutler $\times$ ACBarrie & $\begin{array}{l}\text { Perez-Lara et al., } \\
(2016)\end{array}$ \\
\hline $\begin{array}{l}\text { Flowering } \\
\text { (days) }\end{array}$ & $\begin{array}{l}\text { QFlt.dms- } \\
\quad 7 A .1\end{array}$ & $7 \mathrm{~A}$ & $\begin{array}{l}\text { Tdurum_contig11613_3 } \\
29- \\
\text { wsnp_Ex_c30239_3917 } \\
9460\end{array}$ & RIL & 158 & Cutler $\times$ ACBarrie & $\begin{array}{l}\text { Perez-Lara et al., } \\
\text { (2016) }\end{array}$ \\
\hline $\begin{array}{l}\text { Flowering } \\
\text { (days) }\end{array}$ & $\begin{array}{l}\text { QFlt.dms- } \\
\quad 7 A .1\end{array}$ & $7 \mathrm{~A}$ & $\begin{array}{l}\text { Tdurum_contig11613_3 } \\
29- \\
\text { wsnp_Ex_c30239_3917 } \\
9460\end{array}$ & RIL & 158 & Cutler $\times$ ACBarrie & $\begin{array}{l}\text { Perez-Lara et al., } \\
\text { (2016) }\end{array}$ \\
\hline $\begin{array}{l}\text { Flowering } \\
\text { (days) }\end{array}$ & QFlt.dms-7A & $7 \mathrm{~A}$ & $\begin{array}{l}\text { Tdurum_contig11613_3 } \\
29- \\
\text { wsnp_Ex_c30239_3917 } \\
9460\end{array}$ & RIL & 158 & Cutler $\times$ ACBarrie & $\begin{array}{l}\text { Perez-Lara et al., } \\
(2016)\end{array}$ \\
\hline $\begin{array}{l}\text { Flowering } \\
\text { (DD) }\end{array}$ & QFlt.dms-7A & $7 \mathrm{~A}$ & $\begin{array}{l}\text { Tdurum_contig11613_3 } \\
29- \\
\text { wsnp_Ex_c30239_3917 } \\
9460\end{array}$ & RIL & 158 & Cutler $\times$ ACBarrie & $\begin{array}{l}\text { Perez-Lara et al., } \\
(2016)\end{array}$ \\
\hline
\end{tabular}




\begin{tabular}{|c|c|c|c|c|c|c|c|}
\hline $\begin{array}{l}\text { Flowering } \\
\text { (DD) }\end{array}$ & $\begin{array}{l}\text { QFlt.dms- } \\
\quad 7 A .1\end{array}$ & $7 \mathrm{~A}$ & $\begin{array}{c}\text { Tdurum_contig11613_3 } \\
29- \\
\text { wsnp_Ex_c30239_3917 } \\
9460\end{array}$ & RIL & 158 & Cutler $\times$ ACBarrie & $\begin{array}{l}\text { Perez-Lara et al., } \\
(2016)\end{array}$ \\
\hline $\begin{array}{l}\text { Flowering } \\
\text { (DD) }\end{array}$ & $\begin{array}{l}\text { QFlt.dms- } \\
\quad 7 A .1\end{array}$ & $7 \mathrm{~A}$ & $\begin{array}{l}\text { Tdurum_contig11613_3 } \\
\text { 29- } \\
\text { wsnp_Ex_c30239_3917 } \\
9460\end{array}$ & RIL & 158 & Cutler $\times$ ACBarrie & $\begin{array}{l}\text { Perez-Lara et al., } \\
(2016)\end{array}$ \\
\hline $\begin{array}{l}\text { Flowering } \\
\text { (days) }\end{array}$ & $\begin{array}{l}\text { QFlt.dms- } \\
\quad 7 A .1\end{array}$ & $7 \mathrm{~A}$ & $\begin{array}{c}\text { wsnp_Ra_c63822_6328 } \\
\text { 8359- } \\
\text { wsnp_BG313770A_Ta_ } \\
2 \_3\end{array}$ & RIL & 158 & Cutler $\times$ ACBarrie & $\begin{array}{l}\text { Perez-Lara et al., } \\
(2016)\end{array}$ \\
\hline $\begin{array}{l}\text { Flowering } \\
\text { (DD) }\end{array}$ & $\begin{array}{l}\text { QFlt.dms- } \\
\quad 7 A .2\end{array}$ & $7 \mathrm{~A}$ & $\begin{array}{c}I A C X 4711- \\
w s n p \_K u \_c 7873 \_13486 \\
065\end{array}$ & RIL & 158 & Cutler $\times$ ACBarrie & $\begin{array}{l}\text { Perez-Lara et al., } \\
(2016)\end{array}$ \\
\hline $\begin{array}{l}\text { Maturity } \\
\text { (days) }\end{array}$ & $\begin{array}{l}\text { QMat.dms- } \\
2 D\end{array}$ & $2 \mathrm{D}$ & $\begin{array}{c}\text { Ppd-Dla- } \\
\text { wsnp_CAP11_c3842_18 } \\
29821\end{array}$ & RIL & 158 & Cutler $\times$ ACBarrie & $\begin{array}{l}\text { Perez-Lara et al., } \\
(2016)\end{array}$ \\
\hline $\begin{array}{l}\text { Maturity } \\
\text { (days) }\end{array}$ & $\begin{array}{l}\text { QMat.dms- } \\
2 D\end{array}$ & $2 \mathrm{D}$ & $\begin{array}{c}\text { Ppd-Dla- } \\
\text { wsnp_CAP11_c3842_18 } \\
29821\end{array}$ & RIL & 158 & Cutler $\times$ ACBarrie & $\begin{array}{l}\text { Perez-Lara et al., } \\
(2016)\end{array}$ \\
\hline $\begin{array}{l}\text { Maturity } \\
\text { (days) }\end{array}$ & $\begin{array}{l}\text { QMat.dms- } \\
2 D\end{array}$ & $2 \mathrm{D}$ & $\begin{array}{c}\text { Ppd-Dla- } \\
\text { wsnp_CAP11_c3842_18 } \\
\text { 29821 }\end{array}$ & RIL & 158 & Cutler $\times$ ACBarrie & $\begin{array}{l}\text { Perez-Lara et al., } \\
(2016)\end{array}$ \\
\hline $\begin{array}{l}\text { Maturity } \\
\text { (DD) }\end{array}$ & $\begin{array}{l}\text { QMat.dms- } \\
\qquad D\end{array}$ & $2 \mathrm{D}$ & $\begin{array}{c}\text { Ppd-Dla- } \\
\text { wsnp_CAP11_c3842_18 } \\
29821\end{array}$ & RIL & 158 & Cutler $\times$ ACBarrie & $\begin{array}{l}\text { Perez-Lara et al., } \\
(2016)\end{array}$ \\
\hline $\begin{array}{l}\text { Maturity } \\
\text { (DD) }\end{array}$ & $\begin{array}{l}\text { QMat.dms- } \\
2 D\end{array}$ & $2 \mathrm{D}$ & $\begin{array}{c}\text { Ppd-Dla- } \\
\text { wsnp_CAP11_c3842_18 } \\
29821\end{array}$ & RIL & 158 & Cutler $\times$ ACBarrie & $\begin{array}{l}\text { Perez-Lara et al., } \\
(2016)\end{array}$ \\
\hline $\begin{array}{l}\text { Maturity } \\
\text { (DD) }\end{array}$ & $\begin{array}{l}\text { QMat.dms- } \\
2 D\end{array}$ & $2 \mathrm{D}$ & $\begin{array}{c}\text { Ppd-Dla- } \\
\text { wsnp_CAP11_c3842_18 }\end{array}$ & RIL & 158 & Cutler $\times$ ACBarrie & $\begin{array}{l}\text { Perez-Lara et al., } \\
(2016)\end{array}$ \\
\hline
\end{tabular}




\begin{tabular}{|c|c|c|c|c|c|c|c|}
\hline & & & 29821 & & & & \\
\hline $\begin{array}{l}\text { Maturity } \\
\text { (days) }\end{array}$ & QMat.dms-3B & $3 B$ & $\begin{array}{l}\text { wsnp_Ku_c210_413608 } \\
\text { - Excalibur_c45968_83 }\end{array}$ & RIL & 158 & Cutler $\times$ ACBarrie & $\begin{array}{l}\text { Perez-Lara et al., } \\
\text { (2016) }\end{array}$ \\
\hline $\begin{array}{l}\text { Maturity } \\
\text { (days) }\end{array}$ & $\begin{array}{l}\text { QMat.dms- } \\
\quad 4 A .1\end{array}$ & $4 \mathrm{~A}$ & $\begin{array}{c}\text { CAP12_rep_c4000_432 } \\
- \\
w s n p \_E x \_c 54453 \_5733 \\
1510\end{array}$ & RIL & 158 & Cutler $\times$ ACBarrie & $\begin{array}{l}\text { Perez-Lara et al., } \\
\text { (2016) }\end{array}$ \\
\hline $\begin{array}{l}\text { Maturity } \\
\text { (days) }\end{array}$ & $\begin{array}{l}\text { QMat.dms- } \\
\quad 4 A .1\end{array}$ & $4 \mathrm{~A}$ & $\begin{array}{c}\text { CAP12_rep_c4000_432 } \\
- \\
\text { wsnp_Ex_c54453_5733 } \\
1510\end{array}$ & RIL & 158 & Cutler $\times$ ACBarrie & $\begin{array}{l}\text { Perez-Lara et al., } \\
\text { (2016) }\end{array}$ \\
\hline $\begin{array}{l}\text { Maturity } \\
\text { (DD) }\end{array}$ & $\begin{array}{l}\text { QMat.dms- } \\
\quad 4 A .1\end{array}$ & $4 \mathrm{~A}$ & $\begin{array}{c}\text { CAP12_rep_c4000_432 } \\
- \\
\text { wsnp_Ex_c54453_5733 } \\
1510\end{array}$ & RIL & 158 & Cutler $\times$ ACBarrie & $\begin{array}{l}\text { Perez-Lara et al., } \\
\text { (2016) }\end{array}$ \\
\hline $\begin{array}{l}\text { Maturity } \\
\text { (DD) }\end{array}$ & $\begin{array}{l}\text { QMat.dms- } \\
\quad 4 A .1\end{array}$ & $4 \mathrm{~A}$ & $\begin{array}{c}\text { CAP12_rep_c4000_432 } \\
- \\
w s n p \_E x \_c 54453 \_5733 \\
1510\end{array}$ & RIL & 158 & Cutler $\times$ ACBarrie & $\begin{array}{l}\text { Perez-Lara et al., } \\
\text { (2016) }\end{array}$ \\
\hline $\begin{array}{l}\text { Maturity } \\
\text { (days) }\end{array}$ & $\begin{array}{l}\text { QMat.dms- } \\
\quad 4 A .2\end{array}$ & $4 \mathrm{~A}$ & $\begin{array}{c}\text { wsnp_Ex_c5690_99943 } \\
\text { O5- } \\
\text { wsnp_Ex_rep_c67799_6 } \\
6488792\end{array}$ & RIL & 158 & Cutler $\times$ ACBarrie & $\begin{array}{l}\text { Perez-Lara et al., } \\
\text { (2016) }\end{array}$ \\
\hline $\begin{array}{l}\text { Maturity } \\
\text { (DD) }\end{array}$ & $\begin{array}{l}\text { QMat.dms- } \\
\quad 4 A .2\end{array}$ & $4 \mathrm{~A}$ & $\begin{array}{c}\text { wsnp_Ex_c5690_99943 } \\
\text { 05- } \\
\text { wsnp_Ex_rep_c67799_6 } \\
6488792\end{array}$ & RIL & 158 & Cutler $\times$ ACBarrie & $\begin{array}{l}\text { Perez-Lara et al., } \\
\text { (2016) }\end{array}$ \\
\hline
\end{tabular}




\begin{tabular}{|c|c|c|c|c|c|c|c|}
\hline $\begin{array}{l}\text { Maturity } \\
\text { (days) }\end{array}$ & $\begin{array}{l}\text { QMat.dms- } \\
\quad 4 A .2\end{array}$ & $4 \mathrm{~A}$ & $\begin{array}{c}\text { Ra_c7973_1185- } \\
\text { wsnp_Ex_c10390_1700 } \\
7929\end{array}$ & RIL & 158 & Cutler $\times$ ACBarrie & $\begin{array}{l}\text { Perez-Lara et al., } \\
\text { (2016) }\end{array}$ \\
\hline $\begin{array}{l}\text { Maturity } \\
\text { (days) }\end{array}$ & $\begin{array}{l}\text { QMat.dms- } \\
\quad 4 A .3\end{array}$ & $4 \mathrm{~A}$ & $\begin{array}{c}\text { GENE-2307_140- } \\
R A C 875 \_c 95150 \_286\end{array}$ & RIL & 158 & Cutler $\times$ ACBarrie & $\begin{array}{l}\text { Perez-Lara et al., } \\
(2016)\end{array}$ \\
\hline $\begin{array}{l}\text { Maturity } \\
\text { (DD) }\end{array}$ & $\begin{array}{l}\text { QMat.dms- } \\
\quad 4 A .3\end{array}$ & $4 \mathrm{~A}$ & $\begin{array}{c}\text { GENE-2307_140- } \\
R A C 875 \_c 95150 \_286\end{array}$ & RIL & 158 & Cutler $\times$ ACBarrie & $\begin{array}{l}\text { Perez-Lara et al., } \\
\text { (2016) }\end{array}$ \\
\hline $\begin{array}{l}\text { Maturity } \\
\text { (days) }\end{array}$ & $\begin{array}{l}\text { QMat.dms- } \\
\quad 4 D .1\end{array}$ & $4 \mathrm{D}$ & $\begin{array}{l}\text { Excalibur_c5010_1336- } \\
\text { Kukri_rep_c68594_530 }\end{array}$ & RIL & 158 & Cutler $\times$ ACBarrie & $\begin{array}{l}\text { Perez-Lara et al., } \\
(2016)\end{array}$ \\
\hline $\begin{array}{l}\text { Maturity } \\
\text { (days) }\end{array}$ & $\begin{array}{l}\text { QMat.dms- } \\
\quad 4 D .2\end{array}$ & 4D & $\begin{array}{c}\text { Kukri_rep_c68594_530- } \\
R h t-D 1 b\end{array}$ & RIL & 158 & Cutler $\times$ ACBarrie & $\begin{array}{l}\text { Perez-Lara et al., } \\
\text { (2016) }\end{array}$ \\
\hline $\begin{array}{l}\text { Maturity } \\
\quad \text { (DD) }\end{array}$ & $\begin{array}{l}\text { QMat.dms- } \\
\quad 4 D .2\end{array}$ & 4D & $\begin{array}{c}\text { Kukri_rep_c68594_530- } \\
\text { Rht-D1b }\end{array}$ & RIL & 158 & Cutler $\times$ ACBarrie & $\begin{array}{l}\text { Perez-Lara et al., } \\
\text { (2016) }\end{array}$ \\
\hline $\begin{array}{l}\text { Maturity } \\
\text { (days) }\end{array}$ & $\begin{array}{l}\text { QMat.dms- } \\
\quad 4 D .2\end{array}$ & 4D & $\begin{array}{c}\text { Rht }-D 1 b- \\
\text { wsnp_CAP11_c356_280 } \\
910\end{array}$ & RIL & 158 & Cutler $\times$ ACBarrie & $\begin{array}{l}\text { Perez-Lara et al., } \\
\text { (2016) }\end{array}$ \\
\hline $\begin{array}{l}\text { Maturity } \\
\text { (DD) }\end{array}$ & $\begin{array}{l}\text { QMat.dms- } \\
\quad 4 D .2\end{array}$ & $4 \mathrm{D}$ & $\begin{array}{c}\text { Rht }-D 1 b- \\
\text { wsnp_CAP11_c356_280 } \\
910\end{array}$ & RIL & 158 & Cutler $\times$ ACBarrie & $\begin{array}{l}\text { Perez-Lara et al., } \\
\text { (2016) }\end{array}$ \\
\hline $\begin{array}{l}\text { Maturity } \\
\text { (days) }\end{array}$ & $\begin{array}{l}\text { QMat.dms- } \\
\quad 4 D .2\end{array}$ & 4D & $\begin{array}{c}\text { Rht-D1b- } \\
\text { wsnp_CAP11_c356_280 } \\
910\end{array}$ & RIL & 158 & Cutler $\times$ ACBarrie & $\begin{array}{l}\text { Perez-Lara et al., } \\
\text { (2016) }\end{array}$ \\
\hline $\begin{array}{l}\text { Maturity } \\
\text { (DD) }\end{array}$ & $\begin{array}{l}\text { QMat.dms- } \\
\quad 4 D .2\end{array}$ & 4D & $\begin{array}{c}\text { Rht-Dlb- } \\
\text { wsnp_CAP11_c356_280 } \\
910\end{array}$ & RIL & 158 & Cutler $\times$ ACBarrie & $\begin{array}{l}\text { Perez-Lara et al., } \\
(2016)\end{array}$ \\
\hline $\begin{array}{l}\text { Maturity } \\
\text { (DD) }\end{array}$ & $\begin{array}{l}\text { QMat.dms- } \\
\quad 4 D .2\end{array}$ & 4D & $\begin{array}{c}\text { Rht-D1b- } \\
\text { wsnp_CAP11_c356_280 }\end{array}$ & RIL & 158 & Cutler $\times$ ACBarrie & $\begin{array}{l}\text { Perez-Lara et al., } \\
\text { (2016) }\end{array}$ \\
\hline
\end{tabular}




\begin{tabular}{|c|c|c|c|c|c|c|c|}
\hline & & & 910 & & & & \\
\hline $\begin{array}{l}\text { Maturity } \\
\text { (DD) }\end{array}$ & QMat.dms-5A & $5 \mathrm{~A}$ & $\begin{array}{c}\text { IACX3911- } \\
\text { BS00077858_51 }\end{array}$ & RIL & 158 & Cutler $\times$ ACBarrie & $\begin{array}{l}\text { Perez-Lara et al., } \\
(2016)\end{array}$ \\
\hline $\begin{array}{l}\text { Maturity } \\
\text { (days) }\end{array}$ & QMat.dms-5A & $5 \mathrm{~A}$ & $\begin{array}{l}\text { BS00077858_51- } \\
\text { BS00067209_51 }\end{array}$ & RIL & 158 & Cutler $\times$ ACBarrie & $\begin{array}{l}\text { Perez-Lara et al., } \\
\text { (2016) }\end{array}$ \\
\hline $\begin{array}{l}\text { Maturity } \\
\text { (DD) }\end{array}$ & $\begin{array}{l}\text { QMat.dms- } \\
\quad 7 A .1\end{array}$ & $7 \mathrm{~A}$ & $\begin{array}{c}\text { wsnp_Ra_c63822_6328 } \\
8359- \\
\text { wsnp_BG313770A_Ta_ } \\
2 \_3\end{array}$ & RIL & 158 & Cutler $\times$ ACBarrie & $\begin{array}{l}\text { Perez-Lara et al., } \\
(2016)\end{array}$ \\
\hline $\begin{array}{l}\text { Maturity } \\
\text { (DD) }\end{array}$ & $\begin{array}{l}\text { QMat.dms- } \\
\quad 7 A .2\end{array}$ & $7 \mathrm{~A}$ & $\begin{array}{c}\text { Tdurum_contig37154_1 } \\
90- \\
\text { RAC875_c14982_577 }\end{array}$ & RIL & 158 & Cutler $\times$ ACBarrie & $\begin{array}{l}\text { Perez-Lara et al., } \\
(2016)\end{array}$ \\
\hline $\begin{array}{l}\text { Maturity } \\
\text { (days) }\end{array}$ & $\begin{array}{l}\text { QMat.dms- } \\
\quad 7 A .2\end{array}$ & $7 \mathrm{~A}$ & $\begin{array}{c}\text { RAC875_c14982_577- } \\
\text { Tdurum_contig20214_2 } \\
79\end{array}$ & RIL & 158 & Cutler $\times$ ACBarrie & $\begin{array}{l}\text { Perez-Lara et al., } \\
(2016)\end{array}$ \\
\hline $\begin{array}{l}\text { Maturity } \\
\text { (days) }\end{array}$ & $\begin{array}{l}\text { QMat.dms- } \\
\quad 7 A .2\end{array}$ & $7 \mathrm{~A}$ & $\begin{array}{c}\text { RAC875_c14982_577- } \\
\text { Tdurum_contig20214_2 } \\
79\end{array}$ & RIL & 158 & Cutler $\times$ ACBarrie & $\begin{array}{l}\text { Perez-Lara et al., } \\
(2016)\end{array}$ \\
\hline Plant height & QPht.dms-4D & $4 \mathrm{D}$ & $\begin{array}{c}\text { Rht-D1b- } \\
\text { wsnp_CAP11_c356_280 } \\
910\end{array}$ & RIL & 158 & Cutler $\times$ ACBarrie & $\begin{array}{l}\text { Perez-Lara et al., } \\
(2016)\end{array}$ \\
\hline Plant height & QPht.dms-4D & $4 \mathrm{D}$ & $\begin{array}{c}\text { Rht-D1b- } \\
\text { wsnp_CAP11_c356_280 } \\
910\end{array}$ & RIL & 158 & Cutler $\times$ ACBarrie & $\begin{array}{l}\text { Perez-Lara et al., } \\
(2016)\end{array}$ \\
\hline Plant height & $Q P h t . d m s-4 D$ & $4 \mathrm{D}$ & $\begin{array}{c}\text { Rht-D1b- } \\
\text { wsnp_CAP11_c356_280 } \\
910\end{array}$ & RIL & 158 & Cutler $\times$ ACBarrie & $\begin{array}{l}\text { Perez-Lara et al., } \\
(2016)\end{array}$ \\
\hline Plant height & QPht.dms-4D & 4D & $\begin{array}{c}\text { Rht-D1b- } \\
\text { wsnp_CAP11_c356_280 } \\
910\end{array}$ & RIL & 158 & Cutler $\times$ ACBarrie & $\begin{array}{l}\text { Perez-Lara et al., } \\
(2016)\end{array}$ \\
\hline
\end{tabular}




\begin{tabular}{|c|c|c|c|c|c|c|c|}
\hline Plant height & QPht.dms-4D & $4 \mathrm{D}$ & $\begin{array}{c}\text { wsnp_CAP11_c356_280 } \\
910\end{array}$ & RIL & 158 & Cutler $\times$ ACBarrie & $\begin{array}{l}\text { Perez-Lara et al., } \\
\text { (2016) }\end{array}$ \\
\hline Plant height & QPht.dms-4D & 4D & $\begin{array}{c}\text { wsnp_CAP11_c356_280 } \\
\text { 910-BS00036421_51 }\end{array}$ & RIL & 158 & Cutler $\times$ ACBarrie & $\begin{array}{l}\text { Perez-Lara et al., } \\
\text { (2016) }\end{array}$ \\
\hline Grain yield & QYld.dms-1B & $1 \mathrm{~B}$ & $\begin{array}{c}\text { Tdurum_contig50988_5 } \\
\text { O0- } \\
\text { wsnp_Ex_c13878_2173 } \\
8866\end{array}$ & RIL & 158 & Cutler $\times$ ACBarrie & $\begin{array}{l}\text { Perez-Lara et al., } \\
\text { (2016) }\end{array}$ \\
\hline Grain yield & QYld.dms-2A & $2 \mathrm{~A}$ & $\begin{array}{c}\text { Tdurum_contig86243_2 } \\
\text { 88- BS00063368_51 }\end{array}$ & RIL & 158 & Cutler $\times$ ACBarrie & $\begin{array}{l}\text { Perez-Lara et al., } \\
(2016)\end{array}$ \\
\hline Grain yield & QYld.dms-2D & $2 \mathrm{D}$ & $\begin{array}{c}\text { Ppd-Dla- } \\
\text { wsnp_CAP11_c3842_18 } \\
29821\end{array}$ & RIL & 158 & Cutler $\times$ ACBarrie & $\begin{array}{l}\text { Perez-Lara et al., } \\
\text { (2016) }\end{array}$ \\
\hline Grain yield & QYld.dms-2D & $2 \mathrm{D}$ & $\begin{array}{c}\text { wsnp_CAP11_c3842_18 } \\
29821\end{array}$ & RIL & 158 & Cutler $\times$ ACBarrie & $\begin{array}{l}\text { Perez-Lara et al., } \\
(2016)\end{array}$ \\
\hline Grain yield & QYld.dms-4D & $4 \mathrm{D}$ & $\begin{array}{c}\text { D_GDEEGVY01C7BQ } \\
U \_446- \\
\text { BobWhite_c20689_427 }\end{array}$ & RIL & 158 & Cutler $\times$ ACBarrie & $\begin{array}{l}\text { Perez-Lara et al., } \\
\text { (2016) }\end{array}$ \\
\hline Grain yield & QYld.dms-5B & $5 B$ & $\begin{array}{l}\text { Excalibur_c30667_102- } \\
\qquad u_{-}+6193 \_821\end{array}$ & RIL & 158 & Cutler $\times$ ACBarrie & $\begin{array}{l}\text { Perez-Lara et al., } \\
\text { (2016) }\end{array}$ \\
\hline Grain yield & QYld.dms-5B & $5 B$ & $\begin{array}{c}\text { Ku_c6193_821- } \\
\text { Tdurum_contig31131_1 } \\
98\end{array}$ & RIL & 158 & Cutler $\times$ ACBarrie & $\begin{array}{l}\text { Perez-Lara et al., } \\
\text { (2016) }\end{array}$ \\
\hline $\begin{array}{c}\text { Thylakoid } \\
\text { membrane } \\
\text { damage } 4 / 7\end{array}$ & $\begin{array}{c}\text { QHttmd.ksu- } \\
6 A\end{array}$ & $6 \mathrm{~A}$ & Xbarc113/AGCTCG347 & RIL & 101 & Karl $92 \times$ Ventnor & Talukder et al., (2014) \\
\hline $\begin{array}{c}\text { Thylakoid } \\
\text { membrane } \\
\text { damage } 4 / 7\end{array}$ & $\begin{array}{c}Q H t t m d . k s u- \\
6 A\end{array}$ & $6 \mathrm{~A}$ & Xbarc113/AGCTCG347 & RIL & 101 & Karl $92 \times$ Ventnor & Talukder et al., (2014) \\
\hline $\begin{array}{c}\text { Chlorophyll } \\
\text { content } 4 / 7\end{array}$ & $\begin{array}{c}\text { QHtscc.ksu- } \\
6 A\end{array}$ & $6 \mathrm{~A}$ & Xbarc113/AGCTCG347 & RIL & 101 & Karl $92 \times$ Ventnor & Talukder et al., (2014) \\
\hline Chlorophyll & QHtscc.ksu- & $6 \mathrm{~A}$ & Xbarc113/AGCTCG347 & RIL & 101 & Karl $92 \times$ Ventnor & Talukder et al., (2014) \\
\hline
\end{tabular}




\begin{tabular}{|c|c|c|c|c|c|c|c|}
\hline content $4 / 7$ & $6 A$ & & & & & & \\
\hline $\begin{array}{c}\text { Thylakoid } \\
\text { membrane } \\
\text { damage } 7 / 10\end{array}$ & $\begin{array}{c}\text { QHttmd.ksu- } \\
7 A\end{array}$ & $7 \mathrm{~A}$ & Xbarc121/barc49 & RIL & 101 & Karl $92 \times$ Ventnor & Talukder et al., (2014) \\
\hline $\begin{array}{c}\text { Thylakoid } \\
\text { membrane } \\
\text { damage } 7 / 10\end{array}$ & $\begin{array}{c}\text { QHttmd.ksu- } \\
7 A\end{array}$ & $7 \mathrm{~A}$ & Xbarc121/barc49 & RIL & 101 & Karl $92 \times$ Ventnor & Talukder et al., (2014) \\
\hline $\begin{array}{c}\text { Thylakoid } \\
\text { membrane } \\
\text { damage } 7 / 10\end{array}$ & $\begin{array}{c}\text { QHttmd.ksu- } \\
7 A\end{array}$ & 7A & Xbarc121/barc49 & RIL & 101 & Karl $92 \times$ Ventnor & Talukder et al., (2014) \\
\hline $\begin{array}{l}\text { Chlorophyll } \\
\text { content } \\
\mathbf{4 / 7 / 1 0}\end{array}$ & $\begin{array}{c}\text { QHtscc.ksu- } \\
7 A\end{array}$ & $7 \mathrm{~A}$ & $\operatorname{Bin} 754 / \operatorname{Bin} 45$ & RIL & 101 & Karl $92 \times$ Ventnor & Talukder et al., (2014) \\
\hline $\begin{array}{c}\text { Chlorophyll } \\
\text { content } \\
4 / 7 / 10\end{array}$ & $\begin{array}{c}\text { QHtscc.ksu- } \\
7 A\end{array}$ & $7 \mathrm{~A}$ & Xbarc121/barc49 & RIL & 101 & Karl $92 \times$ Ventnor & Talukder et al., (2014) \\
\hline $\begin{array}{c}\text { Chlorophyll } \\
\text { content } \\
\mathbf{4 / 7 / 1 0}\end{array}$ & $\begin{array}{c}\text { QHtscc.ksu- } \\
7 A\end{array}$ & $7 \mathrm{~A}$ & Xbarc121/barc49 & RIL & 101 & Karl $92 \times$ Ventnor & Talukder et al., (2014) \\
\hline $\begin{array}{c}\text { Plasma } \\
\text { membrane } \\
\text { damage } 7 / 10\end{array}$ & $\begin{array}{c}\text { QHtpmd.ksu- } \\
7 A\end{array}$ & $7 \mathrm{~A}$ & Xbarc121/barc49 & RIL & 101 & Karl $92 \times$ Ventnor & Talukder et al., (2014) \\
\hline $\begin{array}{c}\text { Plasma } \\
\text { membrane } \\
\text { damage } 7 / 10\end{array}$ & $\begin{array}{c}\text { QHtpmd.ksu- } \\
7 A\end{array}$ & $7 \mathrm{~A}$ & Xbarc121/barc49 & RIL & 101 & Karl $92 \times$ Ventnor & Talukder et al., (2014) \\
\hline Plant height & QPh.cau-1B.1 & 1B & Excalibur_c10111_85 & $\mathrm{DH}$ & 203 & ND3338 × JD6. & Guan et al., (2018) \\
\hline Plant height & QPh.cau-1B.2 & 1B & BS00011892_51 & $\mathrm{DH}$ & 203 & ND3338 × JD6 & Guan et al., (2018) \\
\hline Plant height & QPh.cau-1B.3 & 1B & bar308b & $\mathrm{DH}$ & 203 & ND3338 × JD6. & Guan et al., (2018) \\
\hline Plant height & $\begin{array}{c}\text { QPh.cau- } \\
2 D .2\end{array}$ & $2 \mathrm{D}$ & $g w m 296$ & $\mathrm{DH}$ & 203 & ND3338 × JD6. & Guan et al., (2018) \\
\hline
\end{tabular}




\begin{tabular}{|c|c|c|c|c|c|c|c|}
\hline Plant height & $\begin{array}{l}\text { QPh.cau- } \\
2 D .3\end{array}$ & $2 \mathrm{D}$ & $c f d 53$ & DH & 203 & ND3338 × JD6. & Guan et al., (2018) \\
\hline Plant height & QPh.cau-3A.1 & $3 \mathrm{~A}$ & $\begin{array}{c}\text { Tdurum_contig34075_9 } \\
8\end{array}$ & $\mathrm{DH}$ & 203 & ND3338 × JD6. & Guan et al., (2018) \\
\hline Plant height & QPh.cau-3A.2 & $3 \mathrm{~A}$ & CAP11_c1022_117 & $\mathrm{DH}$ & 203 & ND3338 × JD6. & Guan et al., (2018) \\
\hline Plant height & QPh.cau-3A.3 & $3 \mathrm{~A}$ & RAC875_c38975_411 & $\mathrm{DH}$ & 203 & ND3338 × JD6. & Guan et al., (2018) \\
\hline Plant height & QPh.cau-4B.2 & $4 \mathrm{~B}$ & BS00011338_51 & $\mathrm{DH}$ & 203 & ND3338 × JD6. & Guan et al., (2018) \\
\hline Plant height & $\begin{array}{c}\text { QPh.cau- } \\
\text { 4D.1 }\end{array}$ & $4 \mathrm{D}$ & $R h t 2$ & $\mathrm{DH}$ & 203 & ND3338 × JD6. & Guan et al., (2018) \\
\hline Plant height & $\begin{array}{l}\text { QPh.cau- } \\
\text { 4D.2 }\end{array}$ & $4 \mathrm{D}$ & barc105 & DH & 203 & ND3338 × JD6. & Guan et al., (2018) \\
\hline Plant height & QPh.cau-5A.2 & $5 \mathrm{~A}$ & RAC875_c91464_170 & $\mathrm{DH}$ & 203 & ND3338 × JD6. & Guan et al., (2018) \\
\hline Plant height & QPh.cau-6A.2 & $6 \mathrm{~A}$ & GENE-3659_162 & $\mathrm{DH}$ & 203 & ND3338 × JD6. & Guan et al., (2018) \\
\hline Plant height & QPh.cau-6A.3 & $6 \mathrm{~A}$ & $I A A V 5035$ & $\mathrm{DH}$ & 203 & ND3338 × JD6. & Guan et al., (2018) \\
\hline Plant height & QPh.cau-6D & $6 \mathrm{D}$ & barc 54 & $\mathrm{DH}$ & 203 & ND3338 × JD6. & Guan et al., (2018) \\
\hline
\end{tabular}




\section{Simple sequence repeat}

SSR also called the microsatellite marker, it consist of tandem repeat in DNA sequence such as mono, di, tri, tetra and so on. These tandem repeats found in both prokaryotic and eukaryotic genome (Tautz and Renz 1984; Katti et al., 2001; Toth et al., 2000; Salem et al., 2008). They have another name such as short tandem repeats marker, microsatellites markers and sequence tagged microsatellite (STMS) marker etc. it is hyper variable marker available in nature. The variation in these markers is due to subside the DNA replication, in this there are many tandem repeats of nucleotide may be matching due to excision or addition of DNA (Schlotterer and Tautz 1992). Slippage of DNA strand during replication produces mutation than the point mutation. Microsatellite are differentiate based on unique loci called the polymorphism, it can be analysed with the help of PCR. In this technique primer used without radioactive labeled or flurolabeled or radiolabeled to know diverse group of individual with the help of PCR. This unlabeled primer is used analysis with the help of agarose gel electrophoresis or polyacrylamide gel. The unlabeled or fluorolabed primer significantly enhances the research and also panders the research (Wenz et al., 1998; Kumar and Singh, 2020). SSR or microsatellite is codominant in nature or distinguished to heterozygous from homozygous and they are also highly reproducible due to locus specific. There are many scientists has been described the which one particular marker for heat tolerance.

In conclusion, there are more variation for heat tolerance that is varies on development and growth stage but for wheat, reproduction and grain filling stage is more prone to high temperature. Various plant parts are affected by the cellular responses. Therefore to increase yield in wheat during high temperature we will have to understand the idea about heat tolerance mechanism in plants. Molecular marker in plant breeding to detect QTLs for trait of interest is used. But in this review paper, we have described QTLs for heat tolerance. It seems that this technology reveals about the reliable information without any false report.

\section{References}

Al- Khatib, K. and Paulsen, G.M., 1990. Photosynthesis and productivity during high- temperature stress of wheat genotypes from major world regions. Crop Science, 30(5), pp.1127- 1132.

Anon, S., Fernandez, J.A., Franco, J.A. et al., (2004) Effects of water stress and night temperature preconditioning on water relations and morphological and anatomical changes of Lotus creticus

Blum, A., Shpiler, L., Golan, G. and Mayer, J., 1989. Yield stability and canopy temperature of wheat genotypes under drought-stress. Field Crops Research, 22(4), pp.289-296.

Blum, A., Sullivan, C.Y. and Nguyen, H., 1997. The effect of plant size on wheat response to agents of drought stress. II. Water deficit, heat and ABA. Functional Plant Biology, 24(1), pp.4348.

Bohnert, H.J., Gong, Q., Li, P., and Ma, S. (2006) Unravelling abiotic stress tolerance mechanisms: getting genomics going. Curr. Opin. Plant Biol., 9, 180- 188.

Chowdhury, S.I. and Wardlaw, I.F., 1978. The effect of temperature on kernel development in cereals. Australian Journal of Agricultural Research, 29(2), pp.205-223.

Ferris, R., Ellis, R.H., Wheeler, T.R., and Hadley, P. (1998) Effect of high temperature stress at anthesis on grain yield and biomass of field grown crops 
of wheat. Plant Cell Environ, 34, 67-78.

Fokar, M., Blum, A. and Nguyen, H.T., 1998. Heat tolerance in spring wheat. II. Grain filling. Euphytica, 104(1), pp.9-15.

Guan, P., Lu, L., Jia, L., Kabir, M.R., Zhang, J., Lan, T., Zhao, Y., Xin, M., Hu, Z., Yao, Y. and Ni, Z., 2018. Global QTL analysis identifies genomic regions on chromosomes $4 \mathrm{~A}$ and $4 \mathrm{~B}$ harboring stable loci for yield-related traits across different environments in wheat (Triticum aestivum L.). Frontiers in plant science, 9, p.529.

Hays, D., Mason, E., Do, J.H., Menz, M. and Reynolds et al., M., 2007. Expression quantitative trait loci mapping heat tolerance during reproductive development in wheat (Triticum aestivum). In Wheat production in stressed environments (pp. 373-382). Springer, Dordrecht.

Howarth, C.J. (2005) Genetic improvements of tolerance to high temperature, in Abiotic Stresses: Plant Resistance Through Breeding and Molecular Approaches (eds M. Ashraf and P.J.C. Harris), Howarth Press, Inc., New York, pp. 277-300.

Iba, K., 2002. Acclimative response to temperature stress in higher plants: approaches of gene engineering for temperature tolerance. Annual Review of Plant Biology, 53(1), pp.225-245.

Karim, M.A., Fracheboud, Y., and Stamp, P. (1997) Heat tolerance of maize with reference of some physiological characteristics. Ann. Bangladesh Agri., 7, 27-33.

Kato, K., Miura, H. and Sawada, S., 2000. Mapping QTLs controlling grain yield and its components on chromosome $5 \mathrm{~A}$ of wheat. Theoretical and Applied Genetics, 101(7), pp.1114-1121.

Katti, M.V., Ranjekar, P.K. and Gupta, V.S., 2001. Differential distribution of simple sequence repeats in eukaryotic genome sequences. Molecular biology and evolution, 18(7), pp.1161-1167.

Kumar, A., and Singh, V.P., 2020. Maize genetic diversity: Utilization of molecular markers in genetic diversity. International journal of current microbiology and applied sciences, 9(2).

Kumar, A., Chhaya, R. and Singh, V.P. 2020. Genome mapping: Utilization of molecular markers in genome mapping. International Journal of Chemical Studies 2020; 8(1): 3060-3065

Kumar, A., Chhaya, R., Singh, V.P. and Singh, L., 2020. Exploitation of heterosis for grain yield and quality traits in wheat. Journal of Pharmacognosy and Phytochemistry, 9(2), pp.1465-1468.

Kumar, U., Joshi, A.K., Kumari, M., Paliwal, R., Kumar, S. and Röder, M.S., 2010. Identification of QTLs for stay green trait in wheat (Triticum aestivum L.) in the 'Chirya 3' $\times$ 'Sonalika'population. Euphytica, 174(3), pp.437-445.

Maestri, E., Klueva, N., Perrotta, C., Gulli, M., Nguyen, H.T. and Marmiroli, N., 2002. Molecular genetics of heat tolerance and heat shock proteins in cereals. Plant molecular biology, 48(56), pp.667-681.

Mohammadi, V., Zali, A.A. and Bihamta, M.R., 2008. Mapping QTLs for heat tolerance in wheat. J. Agric. Sci. Technol. Vol. 10: 261-267

Owen, P.C. (1971) Responses of a semi-dwarf wheat to temperatures representing a tropical dry season. II. Extreme temperatures. Exp. Agric., 7, 43-47.

Perez-Lara, E., Semagn, K., Chen, H., Iqbal, M., N'Diaye, A., Kamran, A., Navabi, A., Pozniak, C. and Spaner, D., 2016. QTLs associated with agronomic traits in the Cutler $\times$ AC Barrie spring wheat mapping population using single nucleotide polymorphic markers. PLoS 
One, 11(8).

Porter, J.R., 2005. Rising temperatures are likely to reduce crop yields. Nature, 436(7048), pp.174-174.

Rahman, M.S., Wilson, J.H., and Aitken, V. (1977) Determination of spikelet number in wheat. II. Effect of varying light level on ear development. Aust. J. Agric. Res., 26, 575-581.

Reynolds, M., Foulkes, M.J., Slafer, G.A., Berry, P., Parry, M.A., Snape, J.W. and Angus, W.J., 2009. Raising yield potential in wheat. Journal of experimental botany, 60(7), pp.18991918.

Roff, D.A. (1997) Evolutionary Quantitative Genetics, Chapman \& Hall, New York, NY

Sahu, G.K., Kar, M. and Sabat, S.C., 2002. Electron transport activities of isolated thylakoids from wheat plants grown in salicylic acid. Plant Biology, 4(03), pp.321-328.

Saini, H.S. and Aspinal, D. (1982) Abnormal sporogenesis in wheat (Triticum aestivum L.) induced by short periods of high temperature. Ann. Bot., 49, 835846.

Salem, K.F.M., El-Zanaty, A.M. and Esmail, R.M., 2008. Assessing wheat (Triticum aestivum L.) genetic diversity using morphological characters and microsatellite markers. World Journal of Agricultural Sciences, 4(5), pp.538544.

Sangwan, S., Munjal, R., Ram, K. and Kumar, N., 2019. QTL mapping for morphological and physiological traits in RILs of spring wheat population of WH1021 x WH711. Journal of Environmental Biology, 40(4), pp.674682.

Schlötterer, C. and Tautz, D., 1992. Slippage synthesis of simple sequence DNA. Nucleic acids research, 20(2), pp.211215.
Shah, M.M., Gill, K.S., Baenziger, P.S. et al., (1999) Molecular mapping of loci for agronomic traits on chromosome $3 \mathrm{~A}$ of bread wheat. Crop Sci., 39, 1728-1732.

Smertenko, A., Draber, P., Viklicky, V., and Opatrny, Z. (1997) Heat stress affects the organization of microtubules and cell division in Nicotiana tabacum cells. Plant Cell Environ., 20, 1534-1542.

Stone, P.J. and Nicolas, M.E., 1995. Effect of timing of heat stress during grain filling on two wheat varieties differing in heat tolerance. I. Grain growth. Functional Plant Biology, 22(6), pp.927-934.

Talukder, S.K., Babar, M.A., Vijayalakshmi, K., Poland, J., Prasad, P.V.V., Bowden, R. and Fritz, A., 2014. Mapping QTL for the traits associated with heat tolerance in wheat (Triticum aestivumL.). BMC genetics, 15(1), p.97.

Tautz, D. and Renz, M., 1984. Simple sequences are ubiquitous repetitive components of eukaryotic genomes. Nucleic acids research, 12(10), pp.4127-4138.

Tóth, G., Gáspári, Z. and Jurka, J., 2000. Microsatellites in different eukaryotic genomes: survey and analysis. Genome research, 10(7), pp.967-981.

Tura, H., Edwards, J., Gahlaut, V., Garcia, M., Sznajder, B., Baumann, U., Shahinnia, F., Reynolds, M., Langridge, P., Balyan, H.S. and Gupta, P.K., 2020. QTL analysis and fine mapping of a QTL for yield-related traits in wheat grown in dry and hot environments. Theoretical and Applied Genetics, 133(1), pp.239-257.

Vollenweider, P. and Gunthardt- Goerg, M.S. (2005) Diagnosis of abiotic and biotic stress factors using the visible symptoms in foliage. Environ. Pollut., 137, 455-465.

Wenz, H.M., Robertson, J.M., Menchen, S., Oaks, F., Demorest, D.M., Scheibler, D., Rosenblum, B.B., Wike, C., Gilbert, 
D.A. and Efcavitch, J.W., 1998. Highprecision genotyping by denaturing capillary electrophoresis. Genome research, 8(1), pp.69-80.

Yang, J., Zhang, J., Wang, Z., Zhu, Q. and Wang, W., 2001. Hormonal changes in the grains of rice subjected to water stress during grain filling. Plant Physiology, 127(1), pp.315-323.
Zandipour, M., Hervan, E.M., Azadi, A., Khosroshahli, M. and Etminan, A., 2020. A QTL hot spot region on chromosome 1B for nine important traits under terminal drought stress conditions in wheat. Cereal Research Communications, pp.1-8.

\section{How to cite this article:}

Anand Kumar and Vivudh Pratap Singh. 2020. Wheat Heat Tolerance: Mechanism, Impact and Quantitative Trait Loci Associated with Heat Tolerance. Int.J.Curr.Microbiol.App.Sci. 9(02): 893-914. doi: https://doi.org/10.20546/ijcmas.2020.902.107 